\title{
Facial Rejuvenation Enhancing Cheek Lift
}

\author{
Philippe Bellity, Jonathan Bellity \\ Reconstructive and Plastic Surgery, Clinique Hartmann, Neuilly-sur-Seine, France
}

Supported by recent literature on the signs of aging of the middle and lower face, our clinical research has documented a loss of volume of the deep structural components of the central face and a progressive descent of the nasolabial fat and the jowl fat, leading to facial fragmentation. The signs that appear around the age of 45 to 50 years are well targeted by the mini-invasive technique described here. We focused on refitting the jowl fat and the nasolabial fat associated with cutaneous tightening. The use of absorbable barbed sutures (Quill) led to significant improvements, enabling the fitting of fat on fat. In the past 4 years, 167 operations were performed using this technique. The clinical results were very satisfactory, yielding a natural effect caused by the mobilization and strong fixation of the nasolabial fat and the jowl fat in the direction opposite to their displacement.

Keywords Rhytidoplasty / Minimally invasive surgical procedures / Aging

Received: 3 Oct 2016 • Revised: 22 May 2017 • Accepted: 22 Jun 2017

pISSN: 2234-6163 • elSSN: 2234-6171 • https://doi.org/10.5999/aps.2016.01858 • Arch Plast Surg 2017;44:559-563

\author{
Correspondence: Philippe Bellity \\ Reconstructive and Plastic Surgery, \\ Clinique Hartmann, 26 Boulevard \\ Victor Hugo, 92200 Neuilly-sur-Seine, \\ France \\ Tel: +33-6-966-9699 \\ Fax: +33-17-209-1313 \\ E-mail: phbellity@gmail.com
}

\section{INTRODUCTION}

Several anatomical studies on the description of face compartments have shown [1-6] that the face can be thought of as a puzzle whose elements undergo individual modifications with aging and spatially interact with one another. Some compartments lose their volume, notably the deep structures. This phenomenon collapses the anterior architecture of the face, in a way that can be analogized to a deflated bellow.

The abovementioned studies have also shown that some structures do not decrease in volume with time, such as the nasolabial fat and the jowl fat (Fig. 1). These very anterior and very superficial structures are connected to muscles and belong to the more mobile part of the face. The nasolabial fat is anatomically linked to the zygomatic muscle that crosses in front of the other compartments. The jowl fat is linked to the depressor angulis oris.

Moreover, these 2 fatty structures are denser and have smaller lobules than the deeper fat [6]. They adhere to the dermis but not to the underlying superficial muscular aponeurotic system
(SMAS) and progressively slip down the face, causing the skin to sag. Whether the cutaneous loosening precedes or follows the fat loosening in these 2 areas has not yet been determined. Therefore, we believe that surgical refitting should involve both the skin and the fat.

The signs of aging of the middle and lower face associated with a loss of volume of the deep structural components of the central face are marks of facial fragmentation. Recent anatomical descriptions agree with our clinical and intuitive findings. Further, the aging phenomena of the face evolve with an increase in the variability of the individual anatomical structures. With increasing age, aging-related phenomena become more complex, with increasing skin and platysma loosening, fat atrophy, and fat slip, along with a loss of bony volume [1]. However, various classifications can be used with respect to the stages of aging, such as Baker's clinical description $[7,8]$.

Here, we report an innovative surgical technique for facial rejuvenation based on repositioning the nasolabial fat and the jowl fat. 


\section{Fig. 1. Correction of nasolabial and jowl fat descent}

These fat areas are visible and have initiated a downward displacement.

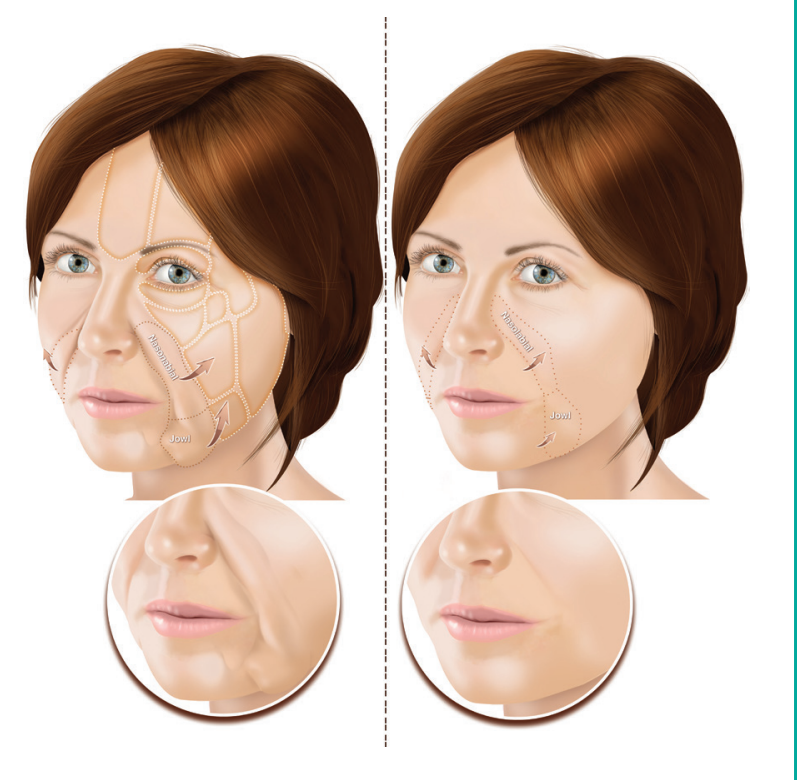

\section{IDEA}

The technique described herein is recommended for patients between 40 and 55 years of age, corresponding to stages 1 and 2 of Baker's classification. In the past 4 years, 167 operations (15 males, 152 females) were performed using this technique. The mean patient age was 53 years (range, 38-65 years), and the mean follow-up duration was 6 months. Patients were examined at 1,3 , and 6 months postoperation.

Before beginning the operation, each patient was positioned in the reverse Trendelenburg supine position, and conventional disinfection methods and sterile drapes were used. A local injection of $1 \%$ lidocaine with 1:100,000 epinephrine $(40 \mathrm{~mL})$, diluted in $250 \mathrm{~mL}$ of saline solution, was administered. Quasi-systematic sedation (performed by an anesthetist) was used considering last-minute stress and a possible increase in the patient's blood pressure, as well as to facilitate greater patient and surgeon comfort.

We made a horizontal or V-shape incision in the sideburn area. An intracapillary oblique extension was added to resect an unsightly cutaneous skin fold if necessary. Next, we made a preauricular vertical incision with a retrolobular extension. We then spread the skin forwards to approach the nasolabial fat and the jowl fat. We ensured that this detachment was delicate and meticulous to avoid ending up deep in the nasolabial structures that are superficial to the SMAS. The nasolabial fat and the jowl fat adhered firmly to the skin; therefore, they had to be suffi-

\section{Fig. 2. Mobilization of nasolabial fat and jowl fat}

The upward and outward mobilization enables a rejuvenation effect.

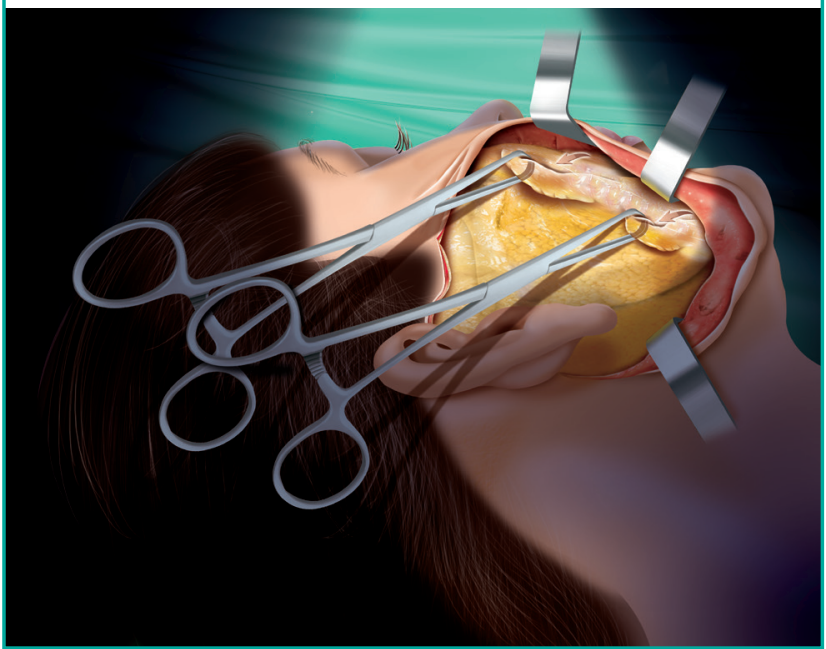

ciently detached to be mobilized and sutured. To do so, the nasolabial fat was approached from the top, as it was easier to approach it from its more medial and thicker part. The mobility of the mobile fat zones was carefully observed by direct traction with Chaput forceps (Fig. 2). The optimal movement was vertical and posterior for the nasolabial fat, and vertical for the jowl fat. The effect was evaluated by the verticalization of the corner of the mouth and by the flattening of the nasolabial furrow. A slight overcorrection was desirable. A very strong X-shaped suture of these 2 structures to the medial cheek fat with a doublearmed Quill suture secured the refitting (Fig. 3). The Quill suture used was a 3/0 absorbable monofilament absorbed completely between 180 and 220 days.

Subcutaneous fixation to the parotid SMAS with padding reduced the dead space and optimized the cutaneous traction [9]. The rise of the cheek fat naturally brought to the top a significant skin flap that exceeded $3 \mathrm{~cm}$. Key cutaneous stitches were executed with 5/0 Monocryl (Polyglécaprone 25, Monofil, Ethicon, Johnson and Johnson Ethicon, USA): The first stitch was made above the tragus and the second at the upper angle of the incision. Cutaneous excision (Fig. 4) was performed when necessary without any tension, both anterior and posterior to the lobule, which allowed us to treat the early signs of cervical loosening. The retrolobular skin, which was nearly cervical, easily moved up. It was firmly attached to the Lore ligament and to the concha, resulting in folds that disappeared in a few weeks.

In some cases, we observed voluminous jowl fat that formed an excessive fat rebound in the lower anterior cheek. Micro-aspiration of the previously marked area is recommended in such circumstances. This localized volume reduction reduced the likelihood of relapse. It was made with a 1-mm micro-cannula 


\section{Fig. 3. X-shaped refitting with barbed sutures}

Barbed sutures allow a better purchase of the tissues and prevent tearing of the fat when it is mobilized.

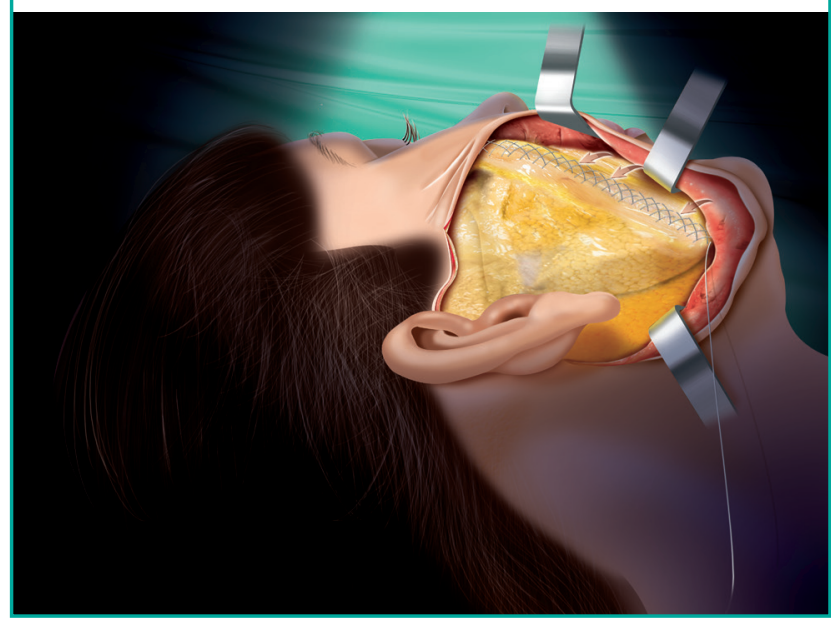

and a $10-\mathrm{mL}$ syringe. Manual depression was applied transcutaneously from an incision at the external limit of the chosen area. This was combined with suction of the fat under the chin. After centrifugation, this fat was re-injected deeply in the tear using a micro-cannula. The procedure lasted approximately 1.5 hours. Monitoring the patient's blood pressure to ensure that it was less than $130 \mathrm{~mm} \mathrm{Hg}$ was important for preventing postoperative bleeding.

Note that a well-made lift of the jowl fat indirectly tightened the adjacent region under the chin. This led to tightening of the neck under the chin, which is difficult to achieve with backwards traction in a traditional cervical lift. Resecting the skin around the earlobe as mentioned earlier enhanced the tightening of the neck laxity towards the back.

\section{Complications}

In this series of 167 patients, 5 hematomas (small to medium in size) occurred, increasing the recovery time of these patients and inducing asymmetrical results for up to 2 months. The time of social inactivity increased from 1-2 to 8 weeks.

Of these 5 hematomas, 1 superficial hematoma had to be surgically evacuated. Another deeper one was responsible for paresis of the upper buccal branch of the facial nerve, as confirmed by ultrasonography and electromyography. We did not evacuate this hematoma. The paresis spontaneously recovered in 8 weeks.

Only 9 simple reoperations had to be performed for imperfections such as a small unappealing bead in the front of the jaw or under the earlobe due to insufficient skin resection.

\section{Clinical applications}

The technique described here is referred to as a "Facial Rejuve-

\section{Fig. 4. Cutaneous resection}

The mean cutaneous resection is $3-4 \mathrm{~cm}$ in front of the ear.

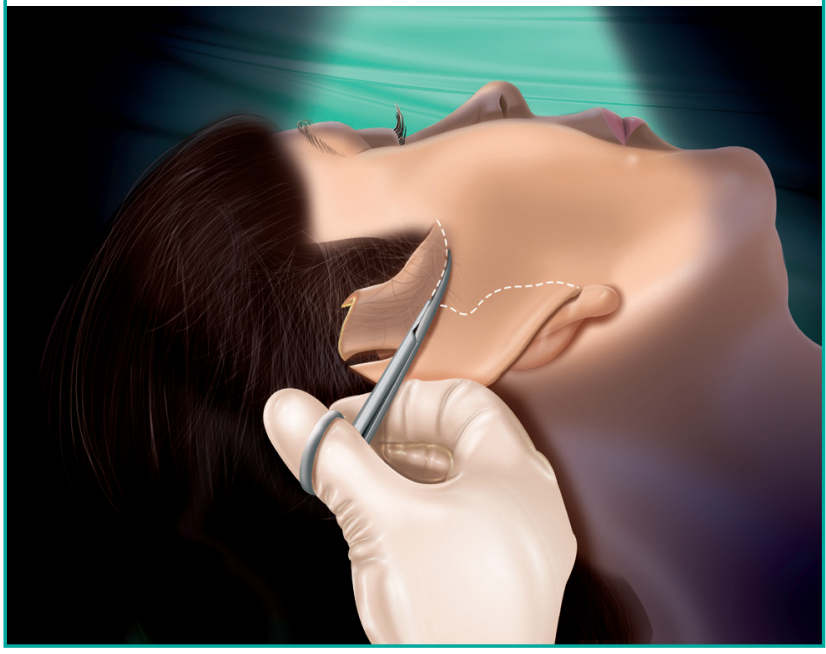

nation Enhancing Cheek lift for enhancing facial rejuvenation (French lift)”. It has several innovative characteristics.

This technique involves the refitting of the cheek and the jowl by mobilizing and fixing the jowl fat and the nasolabial fat in the direction opposite to their displacement and by using a cutaneous resection without tension.

This technique can be performed in women aged between 40 and 55 years, who do not need an cervical-facial lift a priori, but instead require jugal surgery.

An interesting tightening of the neck by 2 concomitant effects was observed: vertical traction of the jowl fat that tightened the under-chin area, and skin resection of the peri-lobular ear that tightened the rearmost portion of the neck.

The use of barbed sutures made this technique relatively quick and precise; the to-and-fro $\mathrm{X}$-shaped double running suture on the fat made it more resistant and less hemorrhagic than standard suture. Figs. 5A and 6A show preoperative photos, and Figs. $5 \mathrm{~B}$ and $6 \mathrm{~B}$ show the postoperative results after 6 months.

\section{DISCUSSION}

The mini-lift or mini-invasive technique described here focused on the refitting of the jowl fat and the nasolabial fat associated with cutaneous tightening $[10,11]$. Facial rejuvenation in a young patient is relatively easy and satisfactory [12] because it is well targeted. The clinical observations of the aging of the young patient's cheek supported by recent anatomical studies $[2,5]$ include loss of volume and descent of the mobile tissues, as if an anterior "column" were slipping from the top of the nasolabial furrow to meet the lowest part of the jowl. While simple, wellidentified volume replacements can provide excellent results for 
Fig. 5. (A, B) Preoperative and 6 months postoperative photographs
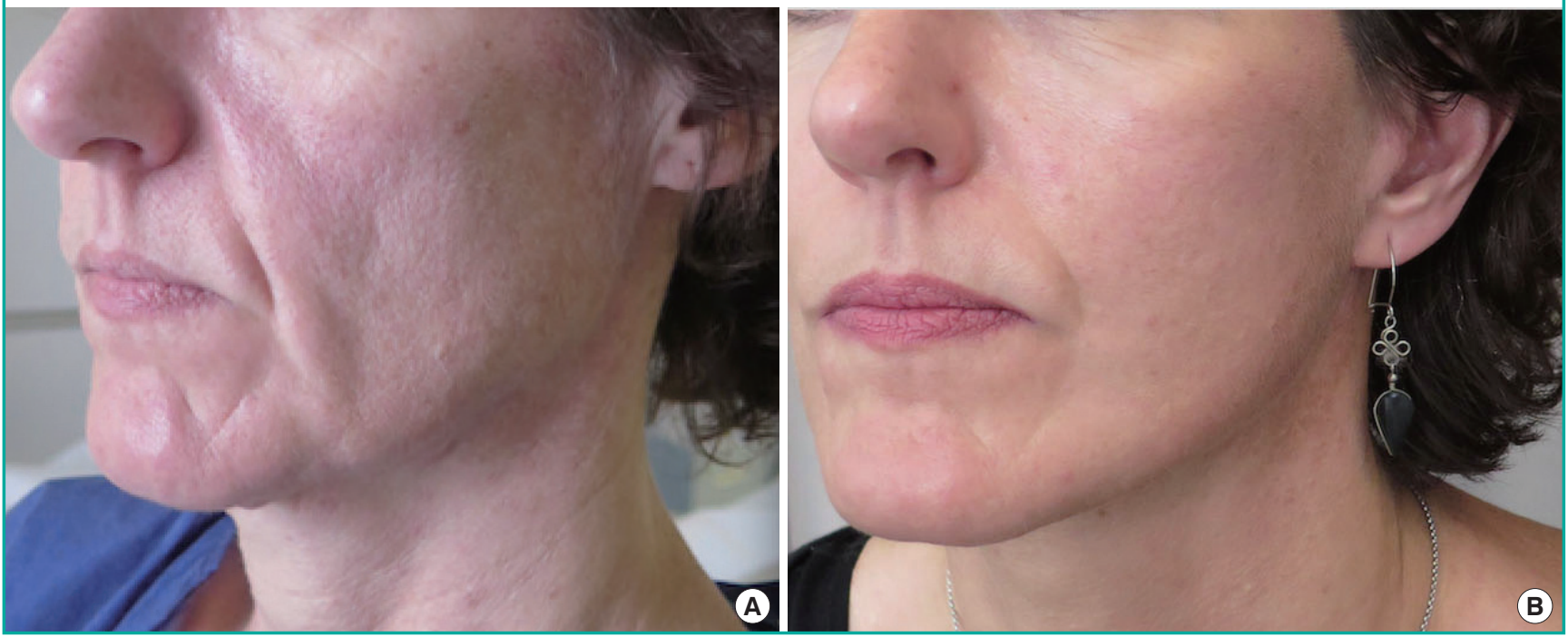

Fig. 6. (A, B) Preoperative and 6 months postoperative photographs
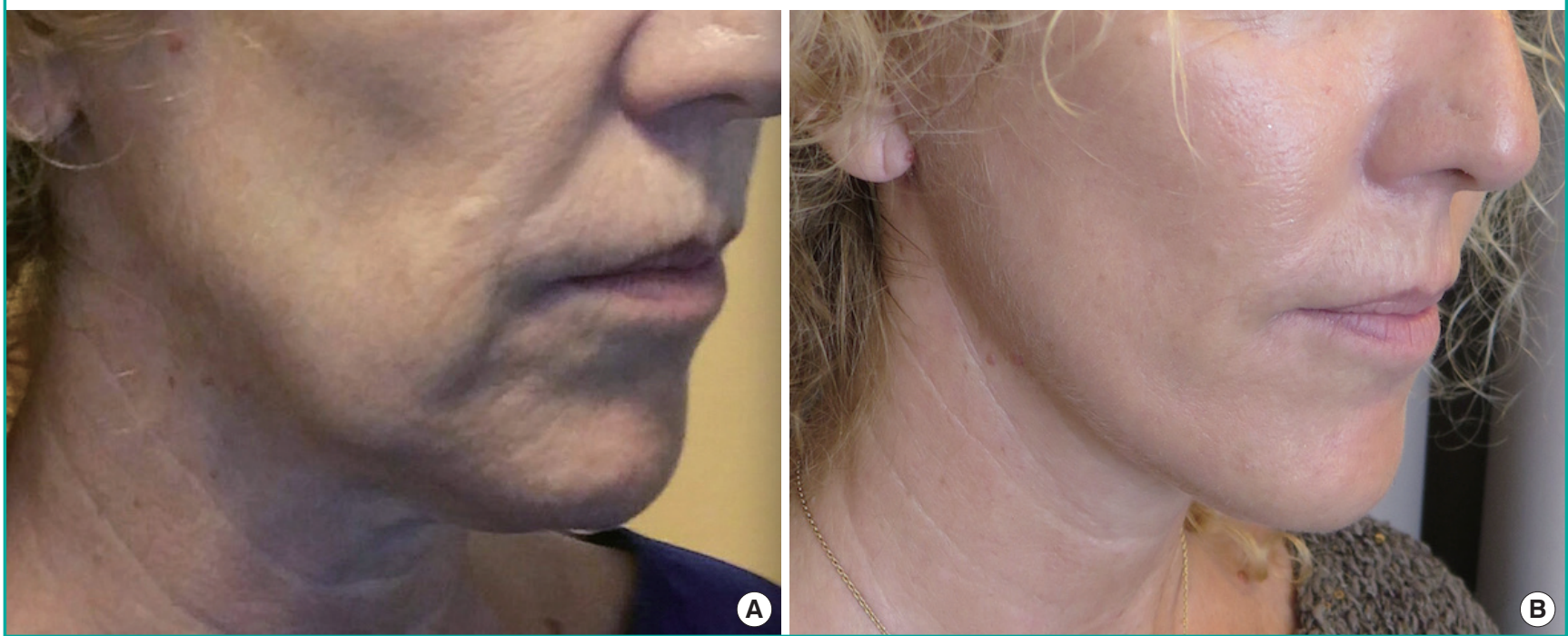

lipofilling or fillers at this stage, the existing medical techniques are inefficient for handling the first signs of ptosis.

This downward movement predicts a possible inverse movement with a refitting of this "column" to restore the shape of this part of the face. In fact, the jowl fat and the nasolabial fat are similar: both tend to slip and are superficial to the SMAS. This movement explains the surgical mobility observed while using this technique. SMAS surgery alone, involving techniques of dissection and plication that have been practiced for years, can certainly improve these marks, but a direct approach to both these fatty structures yields excellent anatomical results at an early stage because of the specific tightening of the displaced tissue. Recent anatomical descriptions confirmed the continuity between these 2 distinct compartments. In terms of surgery, these 2 structures can be pulled up at the same time and sutured relatively high as a mono bloc on the underlying SMAS. Sometimes, these 2 entities are anatomically separated. In such cases, we favor a vertical ascent of the jowl fat first, at which point we assess the mobility of the nasolabial fat. Interestingly, the refitting of the jowl fat involves the horizontality of the corner of the mouth and the ascent of the inferior third of the bitterness fold at the same time.

In this surgery description, the refitting is facilitated by using a Quill suture. This absorbable barbed suture leads to significant improvements [13] that enable the fitting of fat on fat. The complete lack of knots and the self-blocking mobilization at every tissue grip, objectify, and fix the shift immediately. A larger grip on the fat that provides the barbs avoids tearing and the 
'cheese wire' effect. The use of this self-blocking suture allows faster and more effective surgery and leads to a good long-term outcome because of the strength of the suture. The suture is performed in a quick gesture that perfectly compresses the tissues together and allows good hemostasis by compression, as has been observed clinically.

The description of this surgical procedure designed for stages 1 and 2 of Baker's classification offers several other benefits. It is a less invasive technique with a short scar that avoids any incision in the nape of the neck and any useless cervical detachment. In fact, it should be considered a partial jugal facelift meant for the young. This procedure helps to avoid making incisions in the nape, and the cervical detachment reduces inherent complications [14], such as the rather frequent retro-auricular and cervical hematomas, skin necrosis and damage of the great auricular nerve or of the external jugular vein, white or hypertrophic scars on the nape, chronic pain, and distortion of the hairline. This procedure is performed without any tension on the skin, even if the resection is often significant. Moreover, results in the young are more harmonious, with a more natural look, because the lesions are easier to treat owing to the minor displacement. The operation is quick and lasts less than 1.5 hours. Further, the procedure can be performed under local anesthesia; however, the presence of an anesthetist is recommended.

In our opinion, the use of the Quill suture, the padding of the skin, and nightly monitoring to maintain the blood pressure at less than $130 \mathrm{~mm} \mathrm{Hg}$ can explain the scarcity of bleeding events. The postoperative outcomes are quick and simple. It is only necessary to refrain from social activities for approximately $1-2$ weeks, and the patients generally stay in the hospital the night following surgery. Natural-looking results are the rule. Most patients reported that they regained their "former" face. No patient complained of a very tense appearance. Finally, it is easy to combine this technique with blepharoplasty and lipofilling when necessary.

\section{CONFLICT OF INTEREST}

No potential conflict of interest relevant to this article was reported.

\section{PATIENT CONSENT}

The patient provided written informed consent for the publication and the use of their images.

\section{REFERENCES}

1. Richard MJ, Morris C, Deen BF, et al. Analysis of the anatomic changes of the aging facial skeleton using computerassisted tomography. Ophthal Plast Reconstr Surg 2009;25: 382-6.

2. Rohrich RJ, Ghavami A, Constantine FC, et al. Lift-and-fill face lift: integrating the fat compartments. Plast Reconstr Surg 2014; 133:756e-767e.

3. Gierloff M, Stohring C, Buder T, et al. The subcutaneous fat compartments in relation to aesthetically important facial folds and rhytides. J Plast Reconstr Aesthet Surg 2012;65: 1292-7.

4. Gierloff M, Stohring C, Buder T, et al. Aging changes of the midfacial fat compartments: a computed tomographic study. Plast Reconstr Surg 2012;129:263-73.

5. Rohrich RJ, Pessa JE. The fat compartments of the face: anatomy and clinical implications for cosmetic surgery. Plast Reconstr Surg 2007;119:2219-27.

6. Raskin E, Latrenta GS. Why do we age in our cheeks? Aesthet Surg J 2007;27:19-28.

7. Baker DC. Minimal incision rhytidectomy (short scar face lift) with lateral SMASectomy: evolution and application. Aesthet Surg J 2001;21:14-26.

8. Baker DC. Lateral SMASectomy. Plast Reconstr Surg 1997; 100:509-13.

9. Mole B. The double locking face lifting. Ann Chir Plast Esthet 2011;56:15-26.

10. Owsley JQ Fiala TG. Update: lifting the malar fat pad for correction of prominent nasolabial folds. Plast Reconstr Surg 1997;100:715-22.

11. Owsley JQ. Lifting the malar fat pad for correction of prominent nasolabial folds. Plast Reconstr Surg 1993;91:463-74.

12. Jones BM, Marucci DD, Ross GL. Volumetric short scar rhytidectomy: indications, technique and outcomes. J Plast Reconstr Aesthet Surg 2008;61:736-43.

13. Ruff G. Technique and uses for absorbable barbed sutures. Aesthet Surg J 2006;26:620-8.

14. Niamtu J 3rd. Complications in facelift surgery and their prevention. Oral Maxillofac Surg Clin North Am 2009;21: 59-80. 